Alfred K I epsch

\title{
DIE WORTSCHATZBEZIEHUNGEN DES JIDDISCHEN UND DER FRÄNKISCHEN DIALEKTE IM LICHTE DER KULTURGESCHICHTE
}

\section{Einl eit ung}

Im Folgenden wird über lexikalische Transferenzen aus dem Westjiddischen in den ostfränkischen Dialekt berichtet, die bei direkten Erhebungen zu den Mundarten im bayerischen Regierungsbezirk Mittelfranken festgestellt wurden.

In den 1990er J ahren war der Verfasser des vorliegenden Beitrags Explorator für den Sprachatlas von Mittelfranken, eines Teilprojekts des Bayerischen Sprachatlas. Im Verlauf der Aufnahmegespräche fielen immer wieder Spontanbelege für mundartliche Lehnwörter aus dem Hebräischen, die in der allgemeinen deutschen Umgangssprache nicht vorkommen, so z. B. besonders häufig Bore "alte K uh" < hebr. para "K uh", Massik "bösartiges Pferd" < hebr. maziq "Dämon, Quälgeist", Ischa "Geliebte" < hebr. iša "Frau“. In der Nähe des Marktfleckens Schopfloch (vgl. Abschnitt 6) stieg die Zahl der Hebraismen in den Landmundarten exponentiell an und erreichte in diesem ehemaligen "J udendorf" eine Zahl von über 500.

Auf Grund dieser Erfahrungen wurde ein Projekt konzipiert, das parallel zu den Aufnahmen zur Grundmundart anhand von zwei unterschiedlich umfangreichen Fragenkatalogen den jiddischen Lehnwortschatz in den gesprochenen Varietäten Mittelfrankens erhob. Untersucht wurden folgende Varietäten:

1 Die ländlichen Basismundarten. Hierzu wurden mit dem kurzen Fragebogen 86 Gewährspersonen befragt, die zugleich auch an der Grundmundart-Erhebung teilnahmen. W eitere vier Gewährspersonen für die Grundmundart, die in der Nähe von Schopfloch lebten, wurden anhand des ausführlichen $\mathrm{K}$ atalogs befragt.

2 Die Ungangssprache des Nürnberger Ballungsraums. Hierzu wurden mit dem kurzen Fragebogen 14 Gewährsleute aus Nürnberg und Fürth befragt, davon vier aus der älteren und zehn aus jüngeren Generationen. 
3 Die Sondersprache , Lachoudisch“ in Schopfloch. Hierzu wurden mit dem ausführlichen Fragebogen zwölf Personen befragt, davon sieben aus der älteren und fünf aus jüngeren Generationen.

4 Die Sondersprache „) enisch“ in Schillingsfürst. Hierüber gibt es eine Erlanger Dissertation aus der Mitte des 20. J ahrhunderts, ${ }^{1}$ die 1996 durch eine Nacherhebung im Rahmen einer Erlanger Examensarbeit ${ }^{2}$ fortgeschrieben wurde (vgl. Abschnitt 4).

5 Die regionale Viehhändlersprache. Hierzu entstanden anhand des ausführlichen K atalogs vier Aufnahmen (vgl. Abschnitt 5).

6 Das Restjiddische oder "J üdischdeutsche" (vgl. Abschnitt 3). Hierzu konnten noch zwei in Mittelfranken aufgewachsene Angehörige der jüdischen Bevölkerungsgruppe befragt werden. Dies geschah im einen $\mathrm{Fall}$ mit dem ausführlichen $\mathrm{K}$ atalog, im anderen mit einem eigens für das J üdischdeutsche zusammengestellten Questionnaire, das jedoch nur einmal zum Einsatz kam.

Die komplizierte Gemengelage dieser regionalen Varietäten ist historisch bedingt und findet sich in ähnlicher Weise im ganzen deutschen Sprachraum wieder.

In den folgenden Abschnitten soll die Geschichte dieser Varietäten kurz skizziert werden.

\section{Das J idd ische}

Die Entstehung des J iddischen liegt im Dunkeln. Es differenzierte sich wahrscheinlich im Verlauf des Hochmittelalters aus dem koterritorialen Deutschen heraus, indem immer mehr lexikalische Elemente aus dem Hebräischen und Aramäischen in die Alltagssprache der J uden integriert wurden.

Das Hebräische der deutschen J uden war lautlich vom Deutschen beeinflusst: Betonung der Paenultima anstatt der Ultima, infolge dessen Endsilbenschwächung, Hebung und Diphthongierung von betonten Langvokalen, K ürzung unbetonter Langvokale, gebietsweise Binnenhochdeutsche Konsonantenschwächung. Man nennt diese Varietät Aschkenasisch, sie wurde überall im jiddischen Sprachgebiet bei Gebet und Liturgie ver-

1 K naus (1955) und $N$ ier haus-K naus (1973).

2 Fuchs (1996). 
wendet. Fast alle Hebraismen des J iddischen sind durch die aschkenasische Aussprache geprägt.

Im Verlauf des Spätmittelalters entstand eine jiddische Schriftsprache, die mit hebräischen Lettern geschrieben wurde und die für die Lexik der nichtsemitischen Komponente andere orthographische Normen entwickelte als für die Wörter hebräischer oder aramäischer Herkunft. Hebräische Wörter wurden unvokalisiert geschrieben, die Markierung der Vokale für die Wörter der deutschen $\mathrm{K}$ omponente ist in der Frühzeit so inkonsequent, dass eine dialektologische Interpretation kaum möglich ist. ${ }^{3}$

Somit bleibt der Ort der Entstehung des Jiddischen strittig. W ein$\mathrm{r}$ eich vermutet inn am R hein ${ }^{4} \mathrm{~K}$ at $\mathrm{z}$ sucht das deutsch-dialektale Vorbild im Ostmitteldeutschen oder Bairischen ${ }^{5}$ und E gger s (1998), S. 81ff., legt sich auf das Bairische fest, wobei er aber im Wesentlichen das Ostjiddische im Blick hat. Mangels aussagekräftiger zeitgenössischer Quellen kann nur von einem späteren Stand, etwa ab der zweiten Hälfte des 18. J hs. zurückgeschlossen werden. Zu dieser Zeit war das J iddische bereits in eine Dialektlandschaft aufgegliedert, deren einzelne Mundarten einerseits Merkmale aufweisen, die sie mit deutschen Mundarten gemeinsam haben, andererseits auch solche, die nur im J iddischen vorkommen.

Am wichtigsten ist die Unterscheidung zwischen Westjiddisch und Ostjiddisch. Im Spätmittelalter siedelten sich J uden in Polen, Russland und im Baltikum an, die bereits eine Form des J iddischen mitbrachten, also in der Mehrzahl wohl aus Deutschland stammten. Sie behielten diese Sprache für die alltägliche Kommunikation bei, erlernten aber auch die slawischen und baltischen Sprachen ihrer Nachbarn. Auf der lexikalischen Ebene ist daher der Hauptunterschied zwischen W est- und Ostjiddisch die Existenz zahlreicher slawischer Lehnwörter im Ostjiddischen.

Auf der lautlichen Ebene ist ein gemeinsames Merkmal aller westjiddischen Dialekte die Monophthongierung von mhd. ei zu einem langen [a:]. Diese Entsprechungsregel gilt im Bereich der hochdeutschen Mundarten im R heinfränkischen und im Ostfränkischen.

Das W estjiddische gliedert sich, abgesehen von einigen Ü bergangsräumen zum Ostjiddischen, in drei Subdialekte, die definiert werden durch die unterschiedlich konsequent durchgeführte Zweite Lautverschiebung, ähnlich den koterritorialen hochdeutschen Mundarten.

\footnotetext{
3 V gl. Timm (1987), S. 202 und 434f. sowie K at z (1986), S. 25.

4 W einr eich (1980), S. 733.

5 V gl. Eggers (1998), S. 67.
} 
Das Untersuchungsgebiet Mittelfranken liegt im Geltungsbereich des Südwestjiddischen, in dem bezüglich der germanischen Verschlusslaute ein oberdeutscher Lautstand herrscht. Darüber hinaus unterliegen im Südwestjiddischen die Verschlußlaute der Binnenhochdeutschen Konsonantenschwächung. Mhd. ie, üe und uo werden hingegen monophthongiert wie im Mitteldeutschen. Quellen aus dem 19. J $\mathrm{h} .{ }^{6}$ belegen, dass mhd. û nicht zu [au], sondern zu [ou] diphthongiert war und dass germanisch $\mathrm{s}$ vor Konsonanten auch im Wortinlaut zu [š] schibilliert war, beides Merkmale, die an das rezente Schwäbische anklingen. Bairische Merkmale, die nicht auch in anderen hochdeutschen Dialekten auftreten, sind im Westjiddischen nicht anzutreffen. Sie kommen aber im Ostjiddischen, insbesondere im Ostjiddischen Polens vor. ${ }^{7}$

W estjiddisch blieb stets unter dem Dach der sich entwickelnden deutschen Standardsprache und wurde schließlich von ihr absorbiert. Im 18. $\mathrm{J}$ h. verebbte die literarische Produktion des Westjiddischen. Am Ende steht eine Reihe von Dramen, die teils das gesprochene W estjiddische als Charakteristikum negativer Figuren geißeln, ${ }^{8}$ teils, wie die M undartkomödien, den Dialekt für humoristische E ffekte nutzt.

Zur letzteren Gruppe ist eine der wichtigsten Quellen für das gesprochene Südwestjiddische die sog. „F ürther Megille“ von $1828 .{ }^{9}$ Die Sprache ist nahezu durchgängig ein dialektales Westjiddisch, das die gleichen Merkmale aufweist, wie die etwa gleichzeitig herausgekommenen antisemitischen Schriften des "Itzig F eitel St er $n$ “, ${ }^{10}$ einer zweiten Quelle für das gesprochene Südwestjiddische, deren anonymer Verfasser mit hoher Wahrscheinlichkeit in Markt Erlbach, ca. 30 km nordwestlich von Fürth lebte. ${ }^{11}$

Dank dieser beiden literarischen Quellen lässt sich der letzte A bschnitt der jiddischen Sprachgeschichte in Franken recht gut rekonstruieren.

\section{D as J üdischdeutsche}

Ab der Mitte des 19. J hs. begann Westjiddisch auch als gesprochene Sprache auszusterben. Dies war eine Folge der J udenemanzipation, die $z$. B. in Bayern 1850 alle Niederlassungs- und Berufsbeschränkungen für

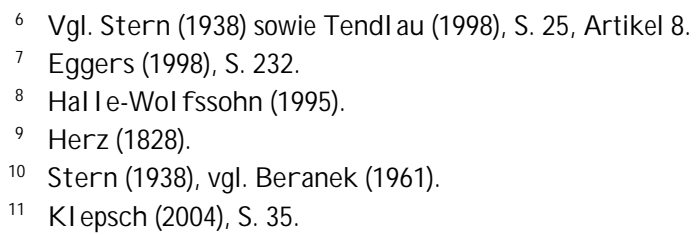


J uden beseitigte. Um 1850 war die einzige größere städtische jüdische Gemeinde im Untersuchungsgebiet Fürth, das „F ränkische J erusalem“ mit 2.650 Personen. ${ }^{12}$ Ansonsten lebten die J uden auf dem Lande, in kleinen Gemeinden mit geringer Basis für materiellen Wohlstand. Die meisten $\mathrm{F}$ amilienväter betätigten sich als $\mathrm{K}$ leinhändler und $\mathrm{H}$ ausierer, der fast ausschließlich von J uden betriebene Viehhandel dürfte einer der lukrativsten Erwerbszweige gewesen sein.

Infolge der Emanzipation setzte eine rapide Landflucht der J uden ein. Bereits um 1900 übertraf die jüdische Bevölkerungszahl Nürnbergs diejenige von Fürth, im J ahr 1925 lebten 81 \% der J uden Mittelfrankens entweder in Nürnberg oder in Fürth. ${ }^{13}$ Auf dem Land waren ganze Gemeinden, die über Synagoge, Friedhof und jüdische Elementarschule verfügt hatten, ausgestorben. In den Städten erfolgte eine kulturelle Assimilation der J uden, insbesondere ein rascher Übergang vom W estjiddischen zur oberdeutschen U mgangssprache. Zwischenstufen sind anhand von zwei Quellen zu beobachten, für die Sprache der Fürther J uden die Gedichte von A. N athan (1905), für das Landjudentum die Wortliste von S. Low enst ein aus dem mittelfränkischen Ort Markt Berolzheim. ${ }^{14}$

Das Ergebnis der Assimilation war eine Sprache, die sich nur durch den Gebrauch von Hebraismen innerhalb der religiösen Domäne von der der Christen unterschied. Der religionsspezifische W ortschatz unterlag in der Lautung einem Wandel hin zu einer für den ganzen aschkenasischen Raum gültigen Konvention, ${ }^{15}$ während die Zahl der im Alltag gebräuchlichen Hebraismen stark zurückging. Wörter wie Pleite, Schlamassel, mies wurden nun aber auch Bestandteil der Umgangssprache von Nichtjuden und sind heute noch in der überregionalen deutschen U mgangssprache zu hören und zu lesen. R egionale U nterschiede gibt es nur noch selten. So gehört B ohei, Buhei "A ufhebens" < hebr. be he "mit überflüssigen Worten "16 eher der (nord)deutschen, Ezes "Tipps" < hebr. etzoth "Ratschläge" eher der österreichischen U mgangssprache an.

Dieses "Rest-Westjiddisch" wird im vorliegenden Text mit MATRAS ${ }^{17}$ als „J üdischdeutsch“ bezeichnet, im Gegensatz zu älterem Gebrauch dieses Ausdrucks, mit dem früher das J iddische im deutschen Sprachraum gemeint war. Im Untersuchungsgebiet wird neben den erwähnten schrift-

12 Vgl. Endres (1978).

13 Ortschaften-V erzeichnis für den Freistaat Bayern (1928).

14 Lowenst ein (1973-1975).

15 Vgl. Burkhard (1969).

16 W einberg (1973), S. 51 und 66.

17 M at r as (1996), S. 48. 
lichen Quellen aus Mittelfranken das J üdischdeutsche durch die eingangs erwähnten beiden Erhebungen mit Sprechern dieser Varietät dokumentiert.

\section{Das R ot wel sche}

Oftmals wurden Rotwelsch und Jiddisch in der Vergangenheit in einen Topf geworfen. Dies geschah insbesondere mit denjenigen Varietäten des J iddischen, die auch von Christen gesprochen wurden. Im Untersuchungsgebiet sind dies die Viehhändlersprache, die Ber anek in seinem viel beachteten Aufsatz über das J iddische in Franken als „Viehhändlerrotwelsch" bezeichnet ${ }^{18}$ und das Schopflocher Lachoudische, das von Arnold (1962) im Rahmen einer rassehygienischen Untersuchung zu fränkischen Rotwelsch-Inseln erwähnt wird. Auch das populäre Wörterbuch von P hil ipp (1983) über das Schopflocher Lachoudische enthält zahlreiche Einträge, die offenbar aus der Dissertation von Knaus über das Schillingsfürster J enische übernommen wurden. ${ }^{19}$ Diese Lemmata wurden von allen für die Direktbefragung herangezogenen Gewährspersonen abgelehnt.

Es ist eine heikle Frage, in welchem Maße Rotwelsch und Jiddisch Übereinstimmungen zeigen. In der Vergangenheit wurden diese Übereinstimmungen, die lediglich auf dem Gebiet der Lexik bestehen, $m$. M. nach aus ideologischen Gründen überbetont.

Das Rotwelsche entstand im Hochmittelalter infolge der Entstehung einer neuen Sozialklasse, nämlich des „F ahrenden Volks“. Dieser Schicht von unbehausten Bettlern, Schaustellern, Prostituierten und Kriminellen gehörten anfangs christlich-deutsche Asoziale, aus der Bahn geworfene Menschen verschiedener Stände an, sie wurde bereichert durch Ausländer gleichen Schicksals und auch durch aus dem sozialen Kontext ihrer Gemeinden und Ghettos ausgebrochene J uden. Hinzu kamen am Ende des Dreißigjährigen Krieges Deserteure aus den Armeen der Kombattantanten und schließlich auch Sinti und Roma, die aus der Organisation ihrer Stämme und Sippen herausgegangen waren oder herausgestoßen worden waren.

Mit dem Exonym Rotwelsch wird eine Gruppe von sondersprachlichen Varietäten auf Basis des Deutschen bezeichnet. Der Sprachname ist be-

18 Ber anek (1961), S. 272.

19 Vgl. K I epsch (2004), S. 22. 
reits zu einem sehr frühen Zeitpunkt belegt, nämlich in der Handschrift des Heidelberger Passionals aus der ersten $\mathrm{Hälfte}$ des 14. J hs. als rot walsch. ${ }^{20}$ Die ursprünglich niederländische Prägung war zunächst ein Appellativum, bestehend aus den Elementen rot "faul, schmutzig" und walsch „unverständliche romanische Sprache“. Im oberdeutschen Raum wurde das Kompositum dann im Sinn von "betrügerische R ede" verwendet und geht im 15. J ahrhundert zum Proprium der Gaunersprache über.

Der erste Beleg für das Endonym J enisch stammt aus dem Text „W iener K ellnersprache" von 1714. ${ }^{21}$ W ol f (1956), Spalte 2346 führt das Wort auf zigeunersprachlich džan "wissen“ zurück, das zunächst als Adjektiv „klug, gescheit" ins Rotwelsche integriert wurde. Dieser Deutung schließt sich auch Seebol d ${ }^{22}$ an.

Das Rotwelsche ist im Wesentlichen ein Sonderwortschatz, die Struktur entspricht dem Deutschen. Die Lexik setzt sich zum größten Teil aus willkürlichen, verfremdenden Wortbildungen aus deutschen Morphemen zusammen, wie z. B. Sitzling für "Stuhl“ oder Lackenpatschel für "Ente“, enthält aber auch Lehnwörter aus romanischen Sprachen, z. B. Patriner "Vater", aus dem Jiddischen, z. B. Bossert "Fleisch" < hebr. basar „Fleisch" und aus der Zigeunersprache, z. B. Zaster "Geld" < zig. saster „Eisen“.

Die einzige rotwelsche Sprachinsel in Mittelfranken ist das Ergebnis einer "Peuplierungsmaßnahme“. Der Fürst von H ohenlohe-Schillingsfürst, aus katholischem Hause, jedoch Herrscher über einen vorwiegend von Protestanten bewohnten Duodezstaat, beabsichtigte in der Mitte des 18. J hs. seine R esidenz mit K atholiken zu bevölkern. Er ließ 1757 im süddeutschen Raum und auch im Ausland ein Flugblatt verbreiten, das katholischen Handwerkern, die sich in Schillingsfürst niederließen, Vergünstigungen versprach.

Da sich jedoch nur wenige zünftige Handwerker meldeten und diese auch nach einiger Zeit wieder abwanderten, weil sie in dem kleinen Territorium keine Absatzmöglichkeiten fanden, erfolgte ein zweiter Aufruf, der nur noch das K riterium „katholisch“ forderte. Es kamen nun Angehörige des Fahrenden Volks, in der Mehrzahl wandernde Maurer, die den Ort Frankenheim, das sog. "Schwarze Viertel“ Schillingsfürsts, erbauten. Sie brachten ihre Geheimsprache, das Rotwelsche, mit und ihre Nachfahren verwendeten sie bis ins ausgehende 20. J h. Das J enische Schillingsfürsts

20 Lühr 1996, S. 17.

21 K I uge 1901, S. 176, Quelle LXVII.

22 K I uge/Seebold (1989), S. 340. 
wurde zuerst durch die Erlanger Germanistin E. K naus (1955) beschrieben, aus der Wolf einige Lemmata für sein Rotwelsches Wörterbuch übernahm. Fortgeschrieben wurde diese Arbeit 1996 durch S. F uchs.

U m Schillingsfürst legt sich eine kleine Kontaktzone von etwa $10 \mathrm{~km}$ Radius, innerhalb derer einige jenische Wörter auch von der alteingesessenen Landbevölkerung verwendet und als Bestandteil ihrer Mundart angesehen werden, z. B. Tschai „Frau“ < zigeunersprachlich tšai "Mädchen".

\section{Die V iehhändl er spr ache}

Eine berufständische Varietät des J iddischen, die auch von Christen gesprochen wurde, ist die (Vieh)händlersprache. Über den Zeitpunkt und den Ort ihrer Entstehung gibt es keine Informationen.

Die ältesten Quellen sind Texte und Glossare aus dem frühen 18. J h. Es handelt sich dabei um sog. „Enthüllungsschriften“ zur E rlernung durch den nichtjüdischen Leser, der somit in die Lage versetzt werden sollte, unseriöse Absprachen unter seinen jüdischen Geschäftspartnern zu bemerken. Der zu Beginn nur latente Antisemitismus steigert sich im Verlauf des 19. J hs. Auch „Itzig Feitel Sterns Schriften“ gehören zu dieser Gattung.

Die Viehhändlersprache war, wie das Rotwelsche, ein künstlich geschaffener Sonderwortschatz. Die in religiösen K ontexten tradierten Hebraismen wurden willkürlich semantisch verändert, des weiteren gab es Wortneubildungen auf Basis hebräischer Elemente und anhand hebräischer oder deutscher Wortbildungsmittel und -regeln.

Als Beispiel für die semantischen Veränderungen sei hier das Wort Massik angeführt, dessen hebräisches Etymon maziq lautet und in der Quellsprache "Dämon, böser Geist" bedeutet. In der Viehhändlersprache wird das W ort jedoch für bösartige Pferde, die zum Ausschlagen und BeiBen neigen gebraucht. Das Wort wurde, wohl durch den Kontakt zu den Viehhändlern in letzterer Bedeutung auch in die Mundarten Frankens integriert und dort auch übertragen für "L ausbube" verwendet.

Den Bereich der Wortbildung soll das Beispiel Doufler illustrieren. Dieses substantivische Simplex wird in der Viehhändlersprache für "Vater" gebraucht, dazu gibt es ein Adjektiv doufel, das „alt" bedeutet. Bei den Erhebungen wurde doufel aber auch mit der Bedeutung "katholisch" zu Protokoll gegeben. Für „K atholik“ wurde die K onstruktion „D ouflemu ne" genannt. 
Dies geht zurück auf eine jiddische Wortbildung aus den hebräischen Elementen taphal "belügen" und 'emuna „Treue, Wahrhaftigkeit", woraus sich „Lügenglaube" ergibt.

Hierzu wurde für die protestantische K onfession nach ähnlichem Muster aus hebr. chadasch "neu" und 'emuna Chaddeschemune "Neuer Glaube" gebildet. Während das Adjektiv chadasch im J iddischen als chodesch oder chidesch verwendet wird, ist taphal nicht gebräuchlich. Somit war Douflemune bald nicht mehr motiviert, das Element doufel wurde nun als das Antonym "alt" zu chodesch "neu“ interpretiert und wurde in der Folge Basis neuer W ortbildungen.

Insbesondere zeichnete sich die V iehhändlersprache aber durch ein eigenes Zahlensystem aus, das weder im Hebräischen noch im J iddischen verwendet wird. Es beruht auf den Namen der hebräischen Buchstaben, die seit dem Altertum auch als Zahlzeichen gebraucht wurden, vor allem zur Paginierung von Büchern.

Innerhalb der Viehhändlersprache deutet sich aufgrund schriftlicher Quellen aus dem 19. Jahrhundert und mündlicher Belege aus dem 20. $\mathrm{J} \mathrm{h.}{ }^{23}$ eine Aufspaltung in eine von J uden und eine von Christen gesprochene Varietät an. Letztere zeichnet sich durch die Lautung [kh] statt $[\mathrm{x}]$ für wortanlautendes hebräisches Cheth an, so dass „Protestant" in der jüdischen Varietät Chaddeschemune, in der christlichen Kaddeschemune lautet.

Das Endonym der Viehhändlersprache lautete ursprünglich Loschen kodesch < hebr. leschon hakódesch "die heilige Sprache“. Damit werden im $\mathrm{J}$ iddischen die antiken Sprachen Hebräisch und Aramäisch bezeichnet, in denen die heiligen Schriften Bibel und Talmud tradiert sind. Die Viehhändlersprache hat einen im Vergleich zum Jiddischen höheren Anteil von Lehnwörtern aus diesen Sprachen, daher die Übertragung. Rezent ist eine Unzahl von Verballhornungen dieser Bezeichnung als Endonyme der Viehhändlersprache belegt, so z. B.

L oschenkaudesch aus Nordwestdeutschland: W ein ber g (1973), S. 169.

Lekoudesch aus R exingen bei Horb: $M$ at $r$ as (1989).

Lotekhđisch aus Rappenau in Nordbaden: M eisinger (1902), S. 122.

L ekouderisch aus Ichenhausen bei UIm: K önig (1996), S. 182.

L orischgorisch mündlich aus Rödlas bei Erlangen.

I slekoudisch aus W elbhausen bei U ffenheim: K il ian (1995), S. 11.

Lachoudisch aus Schopfloch.

23 Vgl. M at r as 1996, S. $55 f$. 


\section{Das Lachoudische Schopf I ochs}

Die Marktgemeinde Schopfloch bildet eine Ausnahme unter den "J udendörfern" Frankens. Die Christen im Dorf waren keine begüterten Bauern, sondern besaßen im Regelfall nur sehr kleine Hofstellen, die allein den Lebensunterhalt nicht sicherten. So verlegten sich viele Schopflocher auf saisonale Nebentätigkeiten. Männer und Frauen arbeiteten im Winter als Hausierer, handelten mit Agrarprodukten oder mit Vieh.

Sie teilten damit die Lebensweise der J uden im Dorf, die zu Beginn des 19. J hs. mit 332 Personen etwa ein Viertel der Dorfbevölkerung ausmachten. ${ }^{24} \mathrm{Es}$ entstanden dadurch intensivere Kontakte zwischen J uden und Christen als andernorts.

Eine große Zahl Schopflocher Männer ging auch, wie die Schillingsfürster J enischsprecher, dem Maurerhandwerk nach. Sie bedienten sich, wie die Schopflocher Händler und Hausierer, der Viehhändlersprache, wobei sie mit den J enischsprechern Kontakt hatten und eine Anzahl rotwelscher Wörter in ihr geheimsprachliches Repertoire aufnahmen. Somit waren vier Subvarietäten des Lachoudischen entstanden: Die Händlersprache der J uden, die Händlersprache der Christen, die Geheimsprache der Maurer und das rudimentäre Lachoudische, das der Rest der Dorfbevölkerung sprach und dessen Lexikon deutlich schmaler ist.

Im J ahr 1994 führte der Verfasser des vorliegenden Beitrags eine Befragung zur Basismundart von Schopfloch durch. Es war zu diesem Zeitpunkt zwar bekannt, dass in Schopfloch eine "Geheimsprache" namens "Lachoudisch" gesprochen wird oder wurde: Es gab ein laienhaftes Wörterbuch des Schopflocher Lehrers K. Phil ipp (1983) und der Schopflocher Bürgermeister H. R. H of mann veranstaltete regelmäßig sprachpflegerische K urse und einen "L achoudisch-Stammtisch“.

Was aber nicht bekannt war, war der außerordentlich große U mfang der lachoudischen Sonderlexik und vor allem die Subvarietäten, in die die lokale Sondersprache gegliedert ist. J ede der in der Folge befragten, meist älteren Gewährspersonen, verfügte über ein größeres oder kleineres individuelles Repertoire aus jiddisch-hebräischen und rotwelschen Elementen in unterschiedlichem Mengenverhältnis. Hierbei spielte eindeutig die Berufsgruppe und die Lebensgeschichte der Gewährsleute eine Rolle. Metzger und Viehhändler kannten fast nur Hebraismen, das größte rotwelsche Repertoire wies die Sprache eines Gewährsmanns auf, der als wandernder Maurer gearbeitet hatte.

24 V gl. P hil ipp (1980 und 1983). 
Das Westjiddische war auch in Schopfloch schon seit längerer Zeit ausgestorben. Die meisten J uden wanderten zwischen 1850 und $1930 \mathrm{ab}$, 1933 lebten nur noch 37 Personen israelitischen Glaubens in Schopfloch. Von innen fielen 24 der nationalsozialistischen Ausrottungspolitik zum Opfer.

Ein jüdischer Gewährsmann, der in Schopfloch aufgewachsen war und seit dem Zweiten Weltkrieg in Amerika lebte, konnte sich noch an die Sprache seiner Eltern erinnern, die er Mammeluschen nannte.

Dabei handelte es sich um ein J üdischdeutsch, das in ähnlicher Form auch durch einen jüdischen Gewährsmann aus dem Dorf Georgensgmünd südlich von Nürnberg zu Protokoll gegeben wurde. Mit Lachoudisch hatte dieses Idiom wenig gemein und wies viel weniger Hebraismen auf.

\section{Land mundar ten und Schopf I ocher U ml and}

Die Grundmundarten in Mittelfranken gehören drei verschiedenen oberdeutschen Dialektgruppen an. Im äußersten Südwesten befindet sich ein kleines Areal des Alemannischen, das sich gegen die anderen Mundarten durch die Isoglosse der Verdumpfung von gedehntem mhd. a abgrenzt. W ährend hier nhd. Rad [ra:d] lautet, wird dieses Wort im restlichen USG als [ro:d] gesprochen. In diesem Gebiet liegt z. B. Mönchsroth.

Nördlich schließt sich das Gebiet ostfränkischer Mundarten an. Diese sind in sich nicht homogen und weisen im Süden und Westen deutliche Interferenzen mit dem Alemannischen auf. Neben lexikalischen Elementen betrifft dies insbesondere die Schibillierung von mhd. -st- im Inund Auslaut, z. B. [du bišd] du bist, die Erhaltung von mhd. uo als fallender Diphthong, z. B. [khua] Kuh und die Vokalisierung von auslautendem, unbetonten -en, z. B. [halde] halten oder [hoage] Haken. In diesem Gebiet liegt z. B. Schopfloch.

Östlich einer Linie, die in Nord-Süd-Richtung etwa $10 \mathrm{~km}$ westlich von Fürth durch das ganze USG verläuft, wird ein ostfränkisch-nordbairischer Ü bergangsdialekt gesprochen, der einerseits die nordbairischen "gestürzten" Diphthonge [ei] und [ou] aus mhd. uo und ie enthält, andererseits aber die ostfränkische Monophthongierung von mhd. ei zu [a:]. Die Wörter lieb, gut und breit lauten hier [leib], [goud] und [bra:d].

In alle ländlichen Mundarten sind ausweislich der Mundartwörterbücher aus kleinen Landstädten und eigener, direkter Erhebungen in Weilern und Dörfern auch eine große Zahl von Hebraismen integriert, die zum großen Teil als alte Elemente der Ortsmundarten, nicht als Fremdwörter, empfunden werden. 
Hierbei ist zu unterscheiden zwischen universell verbreiteten Wörtern wie kouscher "sauber, unverdächtig", verstreut und ohne fest umrissenes Areal auftretenden J iddismen, z. B. I scha „F rau, Geliebte“ und solchen, deren Verbreitungsgebiet durch eine klare Isoglosse abgegrenzt werden kann, wie z. B. letz „frech, böse“. ${ }^{25}$ Bei den beiden letzteren Gruppen ist zu beobachten, dass die Zahl und Dichte der Belege nach Südwesten hin zunimmt.

In der Nähe von Schopfloch ist dieser Anstieg exponentiell. Man kann hier von einem "Kontakthof" des Lachoudischen sprechen, in dem ein gutes Drittel der sondersprachlichen Lexik Schopflochs auch zum Inventar der Mundarten gehört. Belegt ist dies v. a. durch die Erhebungen mit dem ausführlichen Fragebuch (vgl. Abschnitt 1 ) in den Orten Lotterhof und Mönchsroth.

Eine zweite, weit weniger markante Kontaktzone umgibt Schillingsfürst. In der Stadt Rothenburg und in den Orten Diebach, Oberöstheim und v. a. Morlitzwinden konnten einige Elemente des J enischen nachgewiesen werden, die ansonsten außerhalb Schillingsfürsts nicht geläufig sind.

Im Folgenden werden an zwei Fallbeispielen unterschiedliche Typen der Integration hebräischer Lehnwörter in die Mundart mit Hilfe von $\mathrm{K}$ arten besprochen. Eingetragen sind auf den $\mathrm{Karten}$ ausschließlich Belege, die von Sprechern der Grundmundart stammen. Die Belege für das Lachoudische, die V iehhändlersprache, das J enische und das J üdischdeutsche wurden nicht berücksichtigt, da es hier nur um die Integration der betroffenen Wörter in die Mundart geht.

\section{Bärches}

Das W ort bezeichnet eine große Mohnsemmel in Zopfform. Die Gebäckart ist aktuell weit verbreitet und trägt motivierte Benennungen wie H efezopf mit M ohn oder Mohnzopf, es gibt aber auch regionale, teil- bis untransparente Bezeichnungen wie Mohnstriezel (Wien) oder Fleßerl (Burgenland). Verstreut ist hierfür mundartlich aber auch Barches, Bärches oder B erches belegt.

Dialektlexikographen aus dem hochdeutschen R aum führen diese vielfach an, wie das Bayerische Wörterbuch, ${ }^{26} \mathrm{~K}$ önig für Bayerisch Schwa-

25 Die Etymologie ist unsicher. Das W ort könnte von hebr. letz "Spötter“ herzuleiten sein, könnte aber auch germanischer Herkunft sein.

26 Bayerisches Wörterbuch, Heft 7, Spalte 1145. 
ben, ${ }^{27}$ W er ner (1964), S. 257, für Bayerisch Franken und in Mittelfranken M aas (1978), S. 73, für Nürnberg, B er thol d (1975), S. 17, für Fürth, Sch ubart (1973), S. 17, für Rothenburg.

Stets wird zu diesen Einträgen angemerkt, dass es sich bei Wort und Sache um etwas spezifisch J üdisches handelt, so z. B. das Bayerische W örterbuch: "(jüdisches) W eißbrot in Zopfform“, W er ner : "J udengebäck [...]", M aas: „Aus dem J udendeutsch in die Mundart eingedrungen. Nicht mehr häufig".

In lexikologischen und wortgeographischen Darstellungen zum Westjiddischen fehlt Bärches ebenfalls selten. Ber anek (1965), Karte 73, und Guggenheim-Grünberg (1973), Karte 46, stellen seine Verbreitung und Lautvarianten kartographisch dar, W einberg (1973), S. 111, bucht es in der Bedeutung "Sabbatweißbrot mit geflochtenem Zopf", Timm (1987), S. 366, findet es ab 1504 bis ins 18. J ahrhundert als birchoss-kuchen belegt und merkt an, dass das deutsche Basiswort im jüngeren Westjiddischen nicht mehr nachzuweisen sei. Sogar im "Kleinen jüdischen Kochbuch“, S. 72, findet sich ein Rezept für Barches.

Für die Sache gibt es im jiddischen Sprachraum allerdings verschiedene Benennungen. Im Raum Frankfurt kommt Tatscher, ${ }^{28}$ im Nordwestjiddischen kommen Challe, ${ }^{29} \mathrm{~K}$ ouletsch ${ }^{30}$ und Striezel vor.

Auf Grund dieser Beleglage dürfte klar sein, dass das Wort Bärches südwestjiddischen Ursprungs ist. Die Etymologie ist allerdings umstritten. Guggenheim-Grünberg (1973), S. 118, zitiert eine Herleitung von "den für die altdeutsche Göttin Berchta geflochtenen W eißbroten“, W einber g (1973), S. 111, denkt an frz. brioche. In der Mehrzahl und wohl auch zutreffend wird aber die hebräische Wortform birkath "gesegnet sei" als E tymon angeführt. Hiermit beginnen mehrere Berachoth, d. h. Segenssprüche, die mit dem Verzehr von Brot verbunden sind, z. B. „Gesegnet sei der Name dessen, der reich macht...", einer Formel, die häufig auf Brotmessern eingraviert war oder "Gesegnet sei, der hervorbringt...", der Beginn des Segens über das Brot.

Unter den befragten Mundartsprechern aus Mittelfranken kannten etwa noch ein Viertel Wort und Sache. Ganz eindeutig zeigt Karte 1 (s. Anhang), dass das Wort vor allem dort noch bekannt ist, wo es früher

\footnotetext{
27 K önig (1996), S. 180.

28 Das W ort wird hergeleitet von frz. tarte mit westmitteldeutschem Diminutiv -che(n) oder von hochdeutsch Datsch, Dotsch „P fannengebäck“.

$29<$ hebr. challa "Hebeopfer".

$30<$ polnisch kolacz "Kuchen“.
} 
jüdische Landgemeinden gab (schwarze K reise), während dort, wo keine J uden gelebt hatten, auch Bärches kein Begriff ist (Fragezeichen ohne K reis).

Seltener sind die umgekehrten Fälle, nämlich „Wort unbekannt, obwohl ehemals jüdische Bevölkerung im Ort" (Fragezeichen im K reis) und „W ort bekannt, obwohl früher keine J uden im Ort lebten“ (Quadrate).

Die Gewährsleute in den mit schwarzem Kreis markierten Orten erinnerten sich häufig noch an kulturgeschichtliche Details. Eine ehemalige Bäckerin aus Baiersdorf im Norden Mittelfrankens merkte an: „F ür die J uden haben wir das am Samstag, für die Christen am Sonntag gebacken", eine Hausfrau und Bäuerin aus Hüttenbach nordöstlich von Nürnberg: „Die mache ich heute noch, aber etwas anders als die J uden. Bei uns ist es ein einfacher Mohnzopf, bei den J uden kam noch ein kleiner Zopf oben drauf."

Auch in den mit Quadrat gekennzeichneten Orten wurden oft zusätzliche Details angegeben. So präzisierte der Gewährsmann aus Morlitzwinden: „Die gibt es auf Beerdigungen, man sagt auch Leichweck dazu“. Eine Gewährsfrau aus Breitenau erinnerte sich: „M ein Vater war Bäcker, der hat solche Brötchen noch gemacht. Sie waren mit Mohn bestreut. Es gab sie zu Taufen, Konfirmationen und Beerdigungen. Mit Juden hat das nichts zu tun."

Bezüglich der Geographie lässt sich eine W est-Ost-Gliederung des Untersuchungsgebiets feststellen. Im Osten ist, wenn überhaupt, das Wort nur in den ehemaligen "J udendörfern" bekannt und dort werden Wort und Sache meist mit Erinnerungen an die früheren jüdischen Nachbarn in Verbindung gebracht. Im Westen dagegen ist Bärches öfter auch dort ein Begriff, wo früher keine J uden lebten. Das Gebäck wurde dort nur zu besonderen Anlässen, insbesondere anlässlich von Familienfeiern hergestellt. Vermutlich hängt diese Entwicklung einerseits mit der größeren Zahl von jüdischen Landgemeinden in Westmittelfranken, andererseits auch mit einem intensiveren und freundschaftlicheren Kontakt der jüdischen und christlichen Bevölkerungsgruppen zusammen. Aus Schopfloch ist z. B. überliefert, dass auch die Christen manche hohen jüdischen Feiertage, wie insbesondere Simchath-Tora, das Fest der Gesetzesfreude, gemeinsam mit den J uden begingen.

Vereinfachend kann gesagt werden, dass Bärches im östlichen Mittelfranken ein Fremdwort, im W esten dagegen ein mundartliches Lehnwort ist. Diese Vermutung wird gestützt durch die Existenz von mundartlichen Wortbildungen zur Basis Bärches, wie Bärchesweckelein oder Bärcheslein 
„kleine, geflochtene Mohnsemmel im Gegensatz zu Bärches, der größeren Variante".

\section{Kuchem}

Eine andere Art der Integration eines hebräischen Lehnworts zeigt die Geographie der Semantik des Substantivs K uchem.

Das W ort geht zurück auf hebräisch chacham, das sowohl als Adjektiv „klug" als auch als Substantiv „kluger Mensch“ verwendet werden kann. Den beiden jüdischen Gewährsleuten bei der Direkterhebung war es als Substantiv mit der Lautung [x:xem], dem jüdischen Gewährsmann aus Schopfloch darüber hinaus auch mit der Lautung [xu:xem] bekannt. Die letztere dürfte noch der im historischen westjiddischen Dialekt entsprechen. ${ }^{31}$

Die Gewährsleute für die Basismundart sprachen Substantiv und Verb als [khu:xum] oder [khu:xem].

Beide jüdischen Gewährsmänner nannten als K ontextbeispiel spontan eine Redensart: $\mathrm{Er}$ ist der Chochem von der M anischtanne. Dies bedeute so viel wie: „er hat den Nagel auf den Kopf getroffen“ oder auch „er ist hier der größte Schlauberger", was den so bezeichneten gelinde als neunmalklug zurechtweist. Die Herkunft der Redensart war beiden Gewährsmännern transparent.

Sie spielt an auf die Familienfeier, die traditionsgemäß am SederA bend, dem Vorabend des Pesach-F estes abgehalten wird. Seder bedeutet "Ordnung" - der Abend verläuft nach einem festgelegten Ritual, das in der Pesach-Haggada, einer aramäischsprachigen Erzählung, schriftlich festgehalten ist. Teil der Seder-Feierlichkeit ist die sog. Manischtanne. Dies ist eine Art Rollenspiel, im Verlauf dessen der jüngste Sohn dem Familienvater vier Fragen stellt. Der Sohn schlüpft dabei in vier verschiedene Rollen. Vier „Personen“ stellen die F ragen: „Der, der nicht zu fragen versteht", "der Einfältige", "der Böse“ und "der Kluge“. Die Frage des Klugen (chacham) lautet: Ma nischtana halaila haze mikol haleloth? "was unterscheidet diese Nacht von allen Nächten?". Nun kann der Vater beginnen, die biblische Geschichte vom Auszug aus Ägypten zu erzählen.

Dem Chochem oder Chuchem haftet somit etwas Musterschülerhaftes an, worin vielleicht der Keim der Pejorisierung liegt, der das Wort vor al-

31 Nathan (1905), S. 50: „Chuchem“. 
lem in der Mundart unterliegt. Vielleicht hat diese aber auch andere Gründe.

Karte $2 \mathrm{im}$ Anhang zeigt Verbreitung und Bedeutung des Mundartwortes K uchem. Die Belege häufen sich im Süden des Untersuchungsgebiets, insbesondere östlich von Schopfloch, wo es (vgl. Karte 1) nur wenige jüdische Gemeinden gab. Im Nordosten Mittelfrankens gibt es nur dort Belege, wo früher auch J uden ansässig waren, jedoch nicht einmal überall: in Adelsdorf, Baiersdorf und F ürth war K uchem unbekannt. Im südlichen Verbreitungsgebiet des Wortes ist geographisch eine halbkreisförmige Abstufung der Semantik zu beobachten.

In der Nähe von Schopfloch herrscht die positive Bedeutung "kluger Mensch" vor, auf der Karte dargestellt durch hohle Quadrate (Mönchsroth, Gerolfingen, Bechhofen, Dennenlohe, W estheim, Meinheim).

In größerer Entfernung vom K ontaktzentrum zeichnet sich zum einen eine immer stärkere Pejorisierung ab: in Lotterhof (grauer Kreis) und in Kleinried charakterisiert man den Kuchem als "schlauen“, in Ehingen (schwarzes Quadrat) als „besserwisserischen“ Menschen. Der Ehinger Gewährsmann, von Beruf Tischler, erläuterte: „Mein Nachbar ist ein Kuchem. Der will mir sogar in mein Handwerk reinreden."

Ebenfalls durch schwarze Quadrate werden Belege für eine noch weiter gehende Pejorisierung angezeigt: In Hürbel, Absberg, Georgensgmünd, Hechlingen und Langenaltheim kannte man zwar das Wort Kuchem, nannte als Bedeutung nur "schlechter, böser Mensch“. Außerdem kommen in der Randzone auch nahezu antonymische Verwendungen vor wie "leichtsinniger Mensch" (schwarzes Dreieck) oder "gutmütiger Mensch" (hohles Dreieck).

Die starken Pejorisierungen ohne das Merkmal "klug“ und die antonymischen Verwendungen haben sicherlich mit der abnehmenden Geläufigkeit des W orts außerhalb des Gebiets mit dem intensiven Kontakt zu den J iddisch-Sprechern zu tun. Die Komponenten "schlau“ oder "besserwisserisch" könnten dagegen noch, wie oben geschildert, mit der bereits vorhandenen leichten Pejorisierung in der Quellsprache zu tun haben.

Insgesamt kann man Kuchem als ein, wenn auch aussterbendes, mundartliches Lehnwort ansprechen, das weit weniger als der von der Bedeutung her konkretere Begriff Bärches an den nachbarlichen Kontakt der Sprecher des J iddischen und der Mundarten gebunden ist. Es besitzt im Süden Mittelfrankens noch ein geschlossenes Verbreitungsgebiet, ist aber an dessen Rändern von semantischer Verflachung bedroht. 


\section{Zusammenf assung}

Überreste des Jiddischen sind im Untersuchungsgebiet Mittelfranken nicht nur in sondersprachlichen Nischen, sondern auch in den Basismundarten des Untersuchungsgebiets anzutreffen. Sie erlauben durch ihre L autung Rückschlüsse auf frühere sprachliche Zustände der Quellsprache und durch ihre Semantik Rückschlüsse auf historische kulturelle und soziale Verhältnisse. Beides ist anhand schriftlicher Quellen kaum möglich, da diese gerade auf dem flachen Land, wo jahrhundertelang der direkte Kontakt zwischen J uden und Christen am intensivsten war, nicht vorliegen. Eine systematische sprachliche Analyse der aus der Zeit um 1830 aus dem Untersuchungsgebiet vorliegenden Texte, die Denkmäler für das gesprochene historische Westjiddische sind, könnte diese Lücke zumindest regional zu schließen helfen.

\section{Verzeichnis der zitierten Liter at ur}

Al t haus, Hans Peter (1964): J üdisch-H essische Sprachbeziehungen. In: Zeitschrift für Mundartforschung 30 (1963/64), S. 104- 156.

Ar nol d, Hermann (1962): Rotwelsch sprechende Bevölkerungsgruppen im Raume Rothenburg 0.T. - Crailsheim. In: Hessische Blätter für Volkskunde 53.

Bayerisches Wörterbuch (2001). BW B. Heft 7. Bank - Partisane. (= Bayerisch-Österreichisches Wörterbuch II. Bayern). München.

B er anek, Franz J . (1961): Die fränkische Landschaft des J iddischen. In: J ahrbuch für Fränkische Landesforschung 21 (=Festschrift Ernst Schwarz II), S. 267-303.

B er anek, Franz J . (1965): Westjiddischer Sprachatlas. Marburg.

Berthol d, Eugen (1975): „Dei hulli alli o!“ oder: Der Fürther J odler. Ein amüsantes Wörterbuch der Fürther Mundart. Fürth

Bur khar d, Hugo (1969): Der Bimberle - Bamberle - Bomberle. Erinnerungen an eine K ehille. Viel Wahres - Anekdoten/Schnurren - Schmonzes/und etwas zum Nachdenken. Eine Dokumentarbetrachtung. Nürnberg.

Endr es, Rudolf (1978): Geschichte der jüdischen Gemeinde Nürnberg - Fürth im 19. und 20. J ahrhundert. In: Frankenland. Sondernummer 1978 „J üdische Gemeinden in Franken 1100 bis $1975^{\prime \prime}$, S. 23-31.

F uchs, Stefanie (1996): Schillingsfürster J enisch - Eine empirische Untersuchung unter Berücksichtigung der historischen und soziologischen Entwicklung des Rotwelschen. Zulassungsarbeit (Computerausdruck). Erlangen.

Guggenheim-Gr ünber g, Florence (1973): J iddisch auf alemannischem Sprachgebiet. 56 Karten zur Sprach- und Sachgeographie. Zürich (= Beiträge zur Geschichte und Volkskunde der J uden in der Schweiz 10).

H al I e-W ol fssohn, Aaron (1995): Leichtsinn und Frömmelei. Ein Familiengemälde in drei Aufzügen. Transkribierter Neudruck derin hebräischen Lettern gesetzten Ausgabe Breslau 1796. Mit einem Nachwort herausgegeben von Gunnar Och und J utta St r auß. St. Ingbert (=K leines Archiv des achtzehnten J ahrhunderts 22). 
H er z, J oseph (1828): Esther oder die belohnte Tugend. Eine Posse in vier Abschnitten in jüdisch-deutscher Mundart. Fürth .

K at Z, Dovid (1986): J osef Herzens Esther. Zentral-Westjiddisch in Fürth. (Aus dem J iddischen übersetzt von H. Süß). In: Nachrichten für den jüdischen Bürger Fürths 1986, S. 24- 27.

K il ian, Ludwig (1995): ...und das Leben geht weiter. J ugenderinnerungen eines fränkischen Bauern 1925-1950. Uffenheim.

K leines J üdisches K ochbuch für die J üdische $K$ üche. Bearbeitet vom Israelitischen F rauenverein Düsseldorf der J üdischen Haushaltungsschule Frankfurt am Main und der J üdischen Kochschule Berlin. Düsseldorf o.] . Reprint Tel Aviv o.] . [Sinai-Verlag und Doronia-Verlag Stuttgart].

K I epsch, Alfred (2004): Westjiddisches Wörterbuch. Auf der Basis dialektologischer Erhebungen in Mittelfranken. Tübingen.

K I uge, Friedrich (1901): Rotwelsch. Quellen und Wortschatz der Gaunersprache und der verwandten Geheimsprachen. I. R otwelsches Quellenbuch. Straßburg.

K I u ge, Friedrich (1989): Etymologisches Wörterbuch der deutschen Sprache. 22. Auflage unter Mithilfe von Max Bürgisser und Bernd Gregor völlig neu bearbeitet von Elmar Seebol d. Berlin, New York [Zitiert als K I uge/Seebol d].

K naus, Edith (1955): Schillingsfürster R otwelsch. Diss. masch. Erlangen.

K önig, Werner (1996): Zur Sprache der J uden in Ichenhausen. Ein Beitrag zur Rekonstruktion des J iddischen in Ichenhausen sowie seiner ehemaligen Funktion in der deutschen dialektalen Alltagssprache. In: Sprachgeschichtliche Untersuchungen zum älteren und neueren Deutsch. Festschrift für Hans Wellmannn zum 60. Geburtstag. Hrsg. von Werner K önig und Lorelies Or t ner . Heidelberg, S. 175-190.

Lowenstein, Steven (1973-1975): Di sheyres hapleyte fun yidish in franken. In: Yidishe sprakh 32 (1973), S. 24-33; 33 (1974), S. 37-45; 34, (1975), S. 37-43.

L üh r, R osemarie (1996): Zum Sprachnamen R otwelsch. In: Siewert 1996, S. 15- 31.

M aas, Herbert (1978): Wou die Hasen Hoosn und die Hosen Huusn haaßn. Dritte, überarbeitete und ergänzte Auflage. Nürnberg.

M at r as, Y aron (1989): „Lekoudesch“: Integration jiddischer Wörter in die Mundart von Rexingen bei Horb. Mit vergleichbarem Material aus Buttenhausen und Münsingen. Hamburg (=Arbeiten zur Mehrsprachigkeit 33/1989).

M at r as, Y aron (1993): Zur Rekonstruktion des jüdischdeutschen Wortschatzes in den Mundarten ehemaliger "J udendörfer" in Südwestdeutschland. In: Zeitschrift für Dialektologie und Linguistik, LVIII. J ahrgang, H eft 3, S. 267-293.

Mat $r$ as, Yaron (1996): Sondersprachliche Hebraismen: Zum semantischen Wandel in der hebräischen Komponente der südwestdeutschen Viehhändlersprache. In: Siewert 1996, S. 43-58.

M eisinger , Othmar (1902): Lothekôlisch. Ein Beitrag zur K enntnis der fränkischen Händlersprache. In: Zeitschrift für hochdeutsche Mundarten III , S. 121-127.

$N$ at han, Alfred (1905): Chalomes - K lane Scherzlich. F ürth.

N ier haus-K naus, Edith (1973): Geheimsprache in Franken - Das Schillingsfürster J enisch. Rothenburg 0.d. Tauber.

Ortschaften-Verzeichnis für den Freistaat Bayern, nach der Volkszählung vom 16. J uni 1925 und dem Gebietsstand vom 1. J anuar 1928 (= Beiträge zur Statistik Bayerns, Heft 109), hrsg. vom Bayerischen Statistischen Landesamt. München.

Phil ipp, Karl (1980): Geschichte des Marktes Schopfloch. Schopfloch. 
P hil ipp, Karl (1983): Lachoudisch. Geheimsprache Schopflochs. 3. Auflage, Dinkelsbühl. Schubar t, Gertrud (1973): Untern R ätlestuere. R othenburger Wörterbuch. Kirchberg/J agst. Siewert, Klaus (1996): Rotwelsch-Dialekte. Symposion Münster 10. bis 12. März 1995. (= Sondersprachenforschung 1). Wiesbaden.

Sprachatlas von Mittelfranken (2003): Band 1. Einführung. Von Alfred K I epsch unter Mitarbeit von S. R eichel, S. A r zber ger , T. Heyse, A. Mang, H. H. M unske, K. Rädl e, S. R igoll , G. Rost, C. Rudisch und C. Schl ichte. Heidelberg.

„St er n, Itzig Feitel“ (1938): Das Schabbes-Gärtle fer unnere Lait. Chittische Meloche vun Itzig Feitel Stern. Eppes ä Rorität Geblumes fern Brautschmuck. 7. Auflage. Nachdruck der 4. Auflage des Schabbes-Gärtle. Neustadt a.d. Aisch.

Tendl au, Abraham (1998): J üdische Sprichwörter und Redensarten. Als Beitrag zur VolksSprach- und Sprichwörter-K unde. Aufgezeichnet aus dem Munde des Volkes und nach dem Sinn und W ort erläutert. Lizenzausgabe für Parkland Verlag, Köln.

Timm, Erika (1987): Graphische und phonische Struktur des Westjiddischen unter besonderer Berücksichtigung der Zeit um 1600. Tübingen(= Hermea. Germanistische Forschungen, Neue Folge Band 52).

W einber g, Werner (1973): Die Reste des J üdischdeutschen. Zweite, erweiterte Auflage. Stuttgart, Berlin, Köln, Mainz (=Studia Delitzschiana Band 12).

W einber g, Werner (1994): Lexikon zum religiösen Wortschatz und Brauchtum der deutschen J uden. Hrsg. von Walter R öl I . Stuttgart.

W ein r eich, Max (1980): History of the Y iddish Language. Chicago, London.

Werner , Otmar (1964): Das Suffix -es/-as in den ostfränkischen Mundarten. Zur Bedeutung von dia- und synchronischer Betrachtungsweise in der Wortbildungslehre. In: Zeitschrift für Mundartforschung 30 (1963/64), S. 227-274.

W ol f , Siegmund A. (1956): Wörterbuch des Rotwelschen. Deutsche Gaunersprache. Mannheim . 


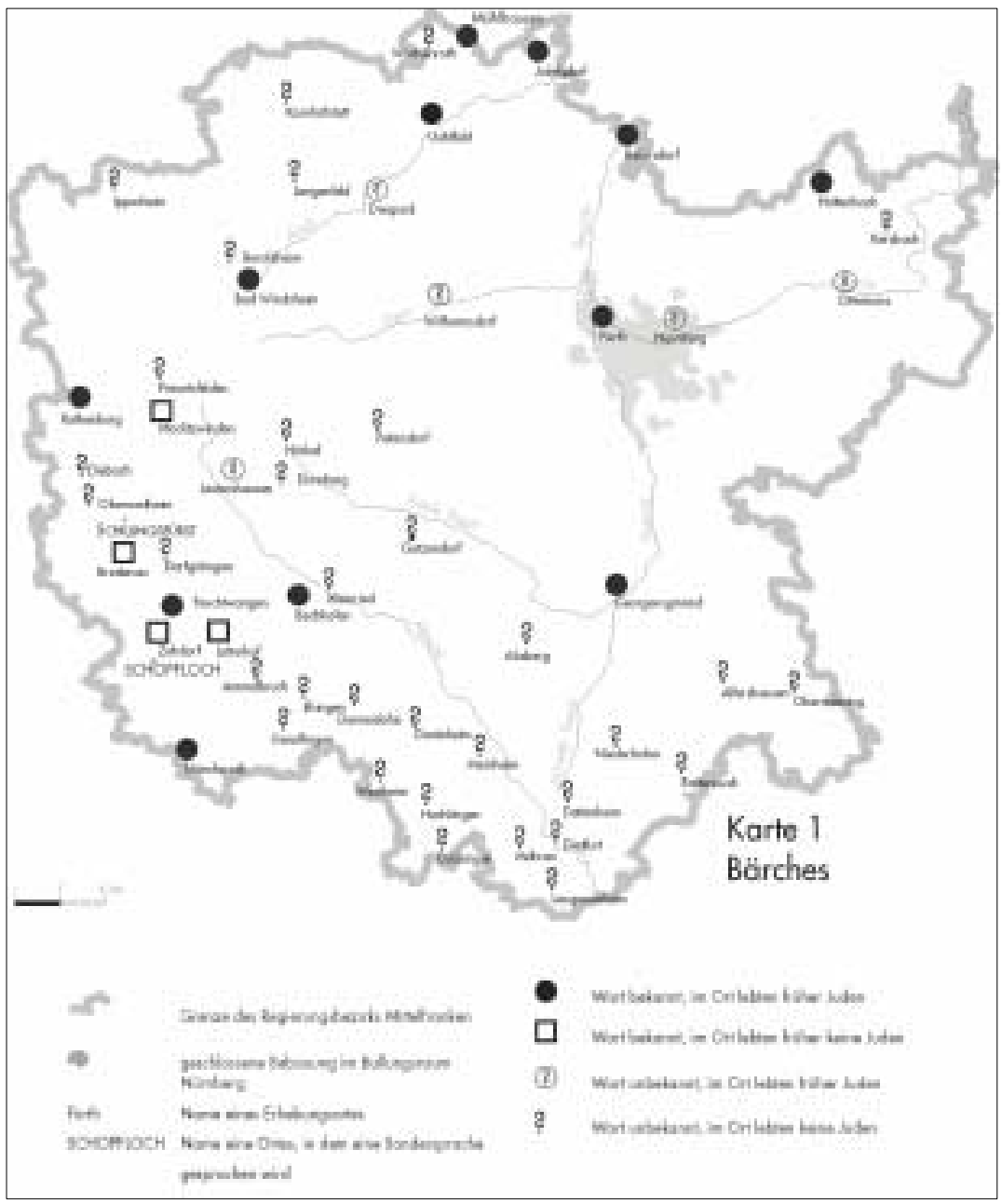

Karte 1: Bärches 


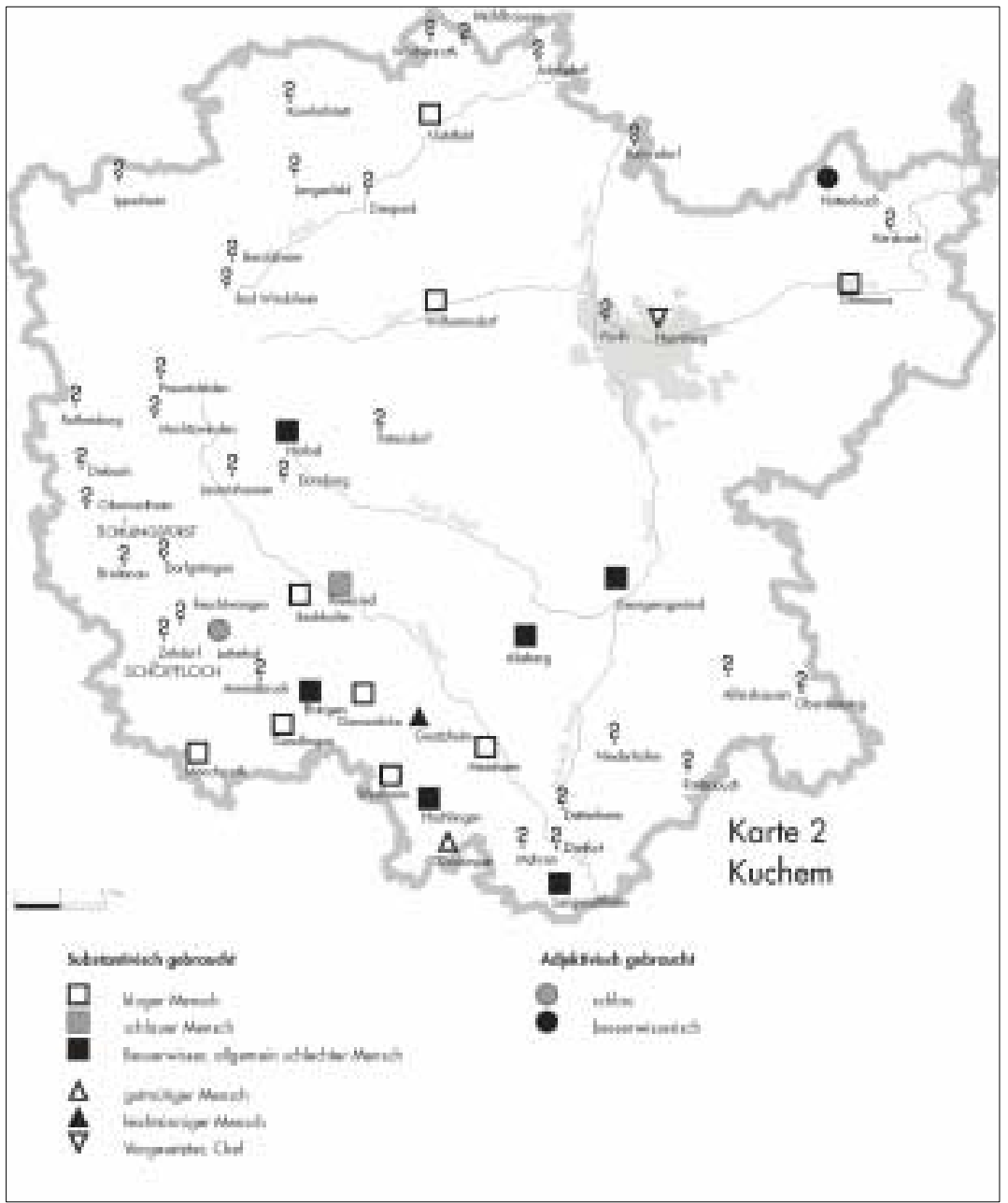

Karte 2: Kuchem 
